\title{
Metasurfaces get a tune up
}

\author{
Two-dimensional structures that are engineered to manipulate electromagnetic radiation are becoming increasingly \\ practical and could be of use in bioelectronics and communications.
}

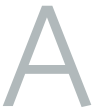
rtificial structures that have been engineered to have unnatural properties are known as metamaterials. The term is typically associated with materials that can manipulate electromagnetic radiation, creating properties such as a negative refractive index or electromagnetic cloaking (not to mention, raising hopes of Harry Potter-esque invisibility cloaks). Such capabilities are achieved via periodic structures with feature sizes smaller than the wavelength of the applied radiation. The structures can be periodic in one, two or three dimensions, and the two-dimensional version, metasurfaces, can now be found in a diverse - and increasingly practical range of device applications.

Metasurfaces can, for instance, be engineered to manipulate electromagnetic fields in, on and around the human body, and in a Review Article in this issue of Nature Electronics, Zhipeng Li, Xi Tian, Cheng-Wei Qiu and John Ho explore the potential applications in bioelectronics and healthcare. The researchers - who are based at the National University of Singapore - first discuss the use of metasurfaces in wireless power transfer, wireless sensing, sensor networks, microwave imaging and magnetic resonance imaging. They then go on to consider the opportunities created by incorporating flexible, stretchable and chemically responsive materials into the systems, as well as the possibilities artificial intelligence offers in optimizing and enhancing designs.

In their Review Article, Ho and colleagues also highlight the emerging importance of creating tunable, reconfigurable and programmable metasurfaces, a topic that is already a focus in applications beyond bioelectronics and healthcare. Such active metasurfaces can be manipulated using a variety of means including electrically, optically, mechanically and thermally - and are of particular interest in wireless communications. Digitally programmable metasurfaces - where ' 0 ' and ' 1 ' are encoded into opposite phase responses of each unit in the metasurface - have, for example, been used to encode signals in both spatial and spectral domains, allowing digital messages to be directly transmitted to different users at different locations simultaneously ${ }^{1}$. Programmable metasurfaces capable of operating at terahertz frequencies have also been created using arrays of complementary metal-oxide-semiconductor (CMOS)-based chip tiles ${ }^{2}$.

Tunable metasurfaces are typically based on materials that require sophisticated and expensive fabrication processes. These processes are also often not compatible with the creation of large-area, flexible systems. This could limit the development of more practical metasurfaces, and the range of applications they can be used in. In an Article elsewhere in this issue, Giorgio Bonacchini and Fiorenzo Omenetto now show that organic electrochemical transistors can be integrated with microwave resonant structures to create tunable metasurfaces. With this approach, both the transistors and the microwave resonators can be fabricated with inkjet printing, which is used to deposit commercial metal nanoparticle and conducting polymer inks onto flexible polyimide substrates.

The electrochemical transistors are based on a mixed ion-electron conducting polymer and can electrically modulate the amplitude and frequency of the microwave resonators. The researchers - who are based at Tufts University - use the technique to build a reconfigurable metasurface composed of 24 electric-LC structures that operate at around $3.8 \mathrm{GHz}$. They also highlight that the approach is compatible with a range of different rigid and flexible substrates, as well as high-throughput printing techniques.

Published online: 23 June 2021

https://doi.org/10.1038/s41928-021-00610-Z

\footnotetext{
References

1. Zhang, L. et al. Nat. Electron. 4, 218-227 (2021).

2. Venkatesh, S., Lu, X., Saeidi, H. \& Sengupta, K. Nat. Electron. 3 , 785-793 (2020)
}

3. Tian, X. et al. Nat. Electron. 2, 243-251 (2019).

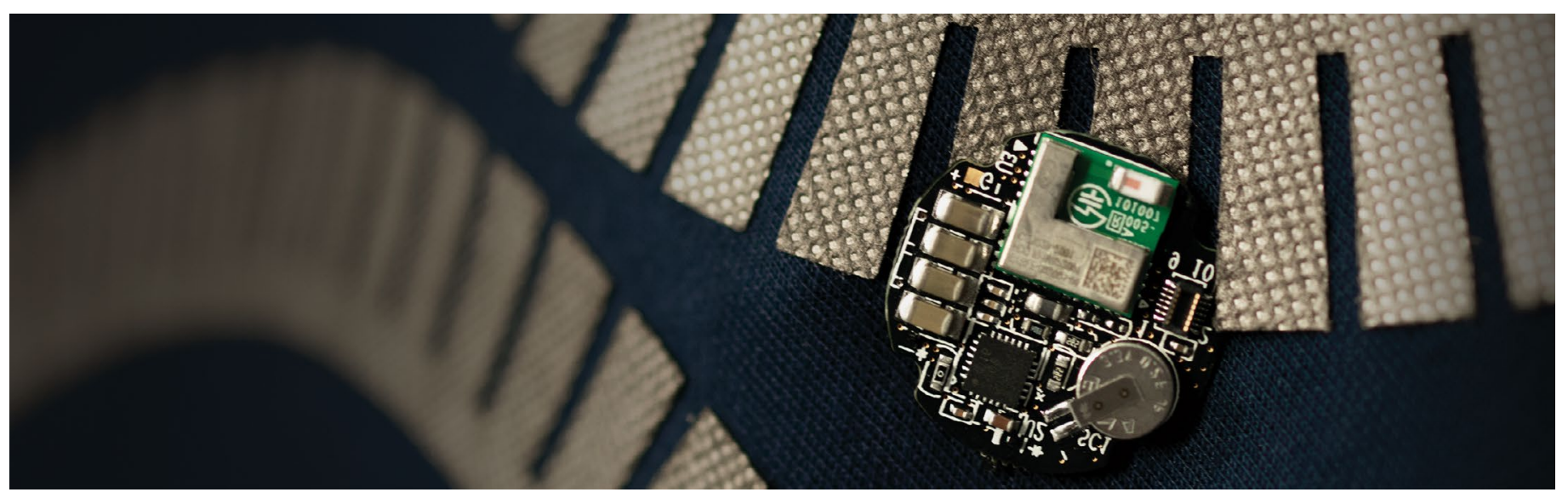

Photograph of a wirelessly powered Bluetooth module that is placed on a metasurface fabricated from a conductive textile. The metasurface can be used to connect wearable sensors around the body ${ }^{3}$. Credit: Xi Tian, National University of Singapore. 